\title{
Gravens By Design: The Case of a NICU with Single- family Rooms: Design Recommendations to Support Family Engagement Behaviors
}

Herminia Machry, PhD, Robert White, MD, Sue Ann Barton, AIA, EDAC, LEED AP

"In NICU environments, evidence shows that while the presence and wellbeing of family members are very important, their active involvement in communication with staff, decision-making, and direct contributions to care is also critical for the development of newborns $(3,4)$."

\section{Introduction}

Saving very low birth weight, preterm, and underdeveloped infants is undoubtedly the primary premise in the neonatal intensive care unit (NICU). Towards this goal, care contributions from family members are integral to facilitate infants' developmental care and vital to support the care continuum at home, after NICU discharge $(1,2)$. In NICU environments, evidence shows that while the presence and wellbeing of family members are very important, their active involvement in communication with staff, decision-making, and direct contributions to care is also critical for the development of newborns $(3,4)$. In a process known as family engagement, healthcare organizations are currently motivated to help reduce NICU length of stay and hospital readmissions by promoting a care partnership between family and staff in the NICU (5). Family Engagement builds on the family-centered care philosophy to activate patients' families as caregivers (6). It is particularly relevant in neonatal care settings due to the great extent to which sick infants rely on their parents during treatment.

The design of NICUs has morphed to include families, initially with the open bay design - room shared by multiple infants with bedside chairs for parents - and more recently with the Singlefamily Room (SFR) design model - private rooms for each family, sometimes including other shared family support spaces(7). In the post-occupancy evaluation of a NICU solely composed of single-family rooms, this study builds on previous research recognizing the current trend of SFRs based on their positive impact on both infant and family outcomes at the $\operatorname{NICU}(8-10)$. We share findings and recommendations around the design of the SFR design model from studying the case of a NICU using SFRs and family support rooms to promote family engagement.

\section{Design as a Resource to Support Family Engagement}

Evidence-based design research shows that the built environment impacts human behavior and health outcomes in healthcare settings (11), such as family engagement. Best practice design in NICUs has been generally guided by clinical evidence, addressing the needs of underdeveloped premature infants in terms of individualized and supportive developmental care, focusing on infant care and parents as infant's support of care (staff and parents care-partners). While the contemporary NICU environment has always been driven by intense medical technology and multidisciplinary staff supervision, individualized developmental care was the first step towards evidence-based design in the NICU, fundamentally defining the ideal environment for infant development as a 'womb-like environment, with controlled lighting, temperature and acoustic conditions(12). Expanding the focus from the illness to the infant's context, supportive developmental care followed by addressing infants' need for parental care as it relates to natural nutrition (breast-milk feeding), touch stimuli (e.g., skin-to-skin care), and sound stimuli (e.g., maternal voice), which closely simulate womb-like conditions while also promoting infant sleep and reducing infant agitation(13,14).

\section{"Expanding the focus from the illness}

to the infant's context, supportive developmental care followed by addressing infants' need for parental care as it relates to natural nutrition (breast-milk feeding), touch stimuli (e.g., skin-to-skin care), and sound stimuli (e.g., maternal voice), which closely simulate womb-like conditions while also promoting infant sleep and reducing infant agitation(13,14)."

Family involvement became an important principle in infant care. Parents were gradually incorporated as part of the NICU environment in response to empirical findings of the benefits of breastmilk feeding and skin-to-skin care to premature infants $(15,16)$. NICU design led to the open bay configuration as a temporary best practice, allowing parents to stay at the infant bedside (7). The open bay, however, raised concerns about infection control,

NEONATOLOGY TODAY is interested in publishing manuscripts from Neonatologists, Fellows, NNPs and those involved in caring for neonates on case studies, research results, hospital news, meeting announcements, and other pertinent topics.

Please submit your manuscript to: LomaLindaPublishingCompany@gmail.com 
privacy, and confidentiality (17), thus provoking discussions in the literature about private rooms (SFRs) being an adequate design strategy for NICUs(18). In the last 25 years, multiple NICUs have been built following the SFR design model, which is likely to become prevalent in the United States, investing extensive resources in planning and construction. This study helps validate this investment by contributing to the expansion and refinement of current family-focused design recommendations about the SFR model in neonatal ICUs, evaluating a NICU intentionally designed to apply the SFR design model as an effort to improve family engagement.

\section{Methods}

We used a qualitative approach to examine a NICU fully embodying the SFR Design model, investigating how different built environment aspects of the unit influence behaviors across four family engagement concepts - family presence, family care, family information exchanges, and family caregiving. We adapted the family involvement model developed by Olding and colleagues(19) to look at behavioral patterns and outcomes across these family engagement concepts, measuring them through the characteristics of all behaviors in which family members are involved during their experiences in the unit. Behavioral characteristics included the type of action or interaction related to family engagement concepts (e.g., daily activities like eating and drinking, and interactions like medical rounds, infant care training, infant feeding, and skin-to-skin care), the type of people involved (e.g., mother, father, nurse, physician), and the number, location, position and movement of people and physical elements during actions/interactions. The built environment was measured by physical characteristics affecting family engagement behaviors at both unit and room levels, including the overall NICU layout (physical arrangement of spaces in the unit), the physical proximity and visibility between spaces, and the physical characteristics within spaces used by families.

\section{Setting}

The case study is a level III NICU located at a Children's Hospital in the Midwestern US. It applies a family-centered care model that includes $24 \mathrm{~h}$ family access with overnight stay and an active family engagement program, which involves various types of family engagement actions and interactions. As depicted in Figure 1, the unit offers two types of SFRs (SFR and Couplet-care SFR), all with adjacent private bathrooms and multiple family support spaces (family-dedicated waiting areas, meeting room, lounge, garden, and atrium).

\section{"The case study is a level III NICU located at a Children's Hospital in the Midwestern US. It applies a family-centered care model that includes $24 \mathrm{~h}$ family access with overnight stay and an active family engagement program, which involves various types of family engagement actions and interactions."}

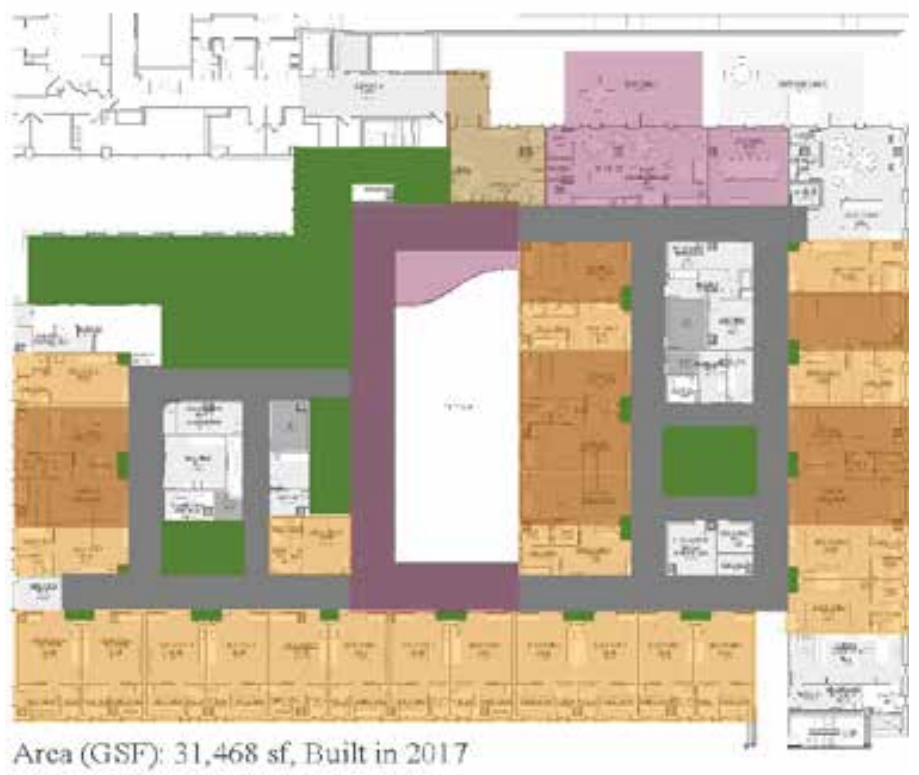

Area (GSF): 31,468 sf, Built in 2017

Total of 30 SFRs

(21 SFRs +9 Couple-carc SFRs)

Single-family rooms (SFR)

Couplet-care SFR

Family entrance

Family support spaces

Corridors

Staff Workstations

Figure 1: Floor plan of the SFR NICU.

\section{Design Process with Family Participation}

Integrating families to participate in infant care was a central focus in the design development of this NICU. Integrated design events with hands-on participation by staff and families were held to develop concepts improving infant, family, and staff-related outcomes and experiences at the unit. Kids and families provided valuable input on many fronts, from the check-in process to the color palettes and environmental graphics developed for the interior design concepts. Mock-up-based design sessions focused on family and staff spaces, where multidisciplinary teams tested layout and equipment needs within the SFR. Refinement of the design was achieved through simulation exercises, where clinical care scenarios were enacted with parents and staff acting in prescribed roles.

\section{DESIgN INTENTIONS FOR FAMILY ENGAGEMENT}

Design sessions resulted in many design decisions intended to encourage and support family engagement. The vision was to create an environment that would provide family accommodation and amenity spaces across the unit and inside patient rooms to facilitate early mother-infant bonding, which is historically limited during the first days of the infant's life in the NICU. The layout of the unit was organized into two neighborhoods around a central courtyard garden overlooking a treehouse to foster a welcoming arrival and provide daylight and natural views for every patient room. A family lounge was located next to the family arrival space and offered families a shared kitchen, eating and activity areas, and direct access to an outdoor garden terrace to provide respite, reduce stress and improve optimism.

Two innovative room prototypes were developed: an enhanced 
SFR (with a window and private toilet with shower) and the Couplet-care SFR. Informed by insights from conversations with NICU leadership at the Karolinska Institute in Sweden, the design of the SFR included these resources. Interview responses were transcribed verbatim and thematically analyzed with an observational, survey, and physical data.

\section{Summary of Findings}

Data from 109 hours of field observations (154 observation logs), 24 semi-structured interviews (9 families and 15 staff members), and 14 responded surveys (29\% response rate) amounted to 517 initial codes related to family engagement behavioral outcomes, and 135 codes about built environment factors impacting family engagement behaviors.

The SFR was the space where most types of actions/interactions occurred. Patterns associating spaces with actions/interactions at the unit showed that while families were using the SFR for most family engagement interactions, support spaces were being used when families prepared their food and drinks, worked on the artwork, and engaged in childcare, distracting their other kids. Family caregiving interactions were unsurprisingly found only in the SFR, whereas family care and information exchanges were associated with both SFRs and the meeting room. The meeting room was utilized for interactions related to family-staff communication and education requiring more people and family focus (e.g., CPR and safe car seat training), where families mainly reported meeting each other in the unit (they met during classes). Staff workstations, on the other hand, were rarely mentioned by families but sometimes mentioned by staff or observed in use when a parent briefly left the SFR to look for staff in areas close to their room; when families greeted staff at the front desk (family access/waiting room); and when family members visited staff in their private offices (e.g., lactation room or social worker office), which were located in corridors connecting SFRs to the family arrival space. Corridors and the family arrival space, in turn, were associated with family-to-family and family-to-staff socialization.

\section{"Observations and interviews helped consolidate functional zones in the SFR (Figure 2). During data collection, families were usually sitting at the kangaroo chair or family bed during all actions and
interactions."}

Observations and interviews helped consolidate functional zones in the SFR (Figure 2). During data collection, families were usually sitting at the kangaroo chair or family bed during all actions and interactions. The staff were usually at the computer area next to the infant bed. Other room areas were either transition areas or areas occasionally shared by staff and family for infant care activities. Family and staff worked together around the information board (information board zone) and at the counter and sink area (feeding care zone), where activities pertained to warming, cleaning, storing, and staging infant milk supplies. Family-staff collaboration also happened on direct infant care activities like holding and cleaning the infant around the infant bed (namely infant zone). It included a "trash zone" (e.g., for dispensing dirty diapers) conveniently accessed by both the inside and the outside of the room.

Built environment characteristics at both room and unit levels emerged as factors impacting family engagement behavioral outcomes. The unit's layout was arranged, so that family support spaces were close to the family entrance and the meeting room, which staff and family perceived as convenient and easily accessible during their flow between activities. Positive feedback came from having family classes within close proximity to infants and having easy access to toys at the family lounge to distract kids accompanying parents during class. Staff perceived the physical proximity between staff offices and the family entrance as supportive to family-staff face-to-face communication, which occurred through informal family visits to these offices. On the other hand, the family thought that the physical proximity between SFRs and staff workstations facilitated their access to staff.

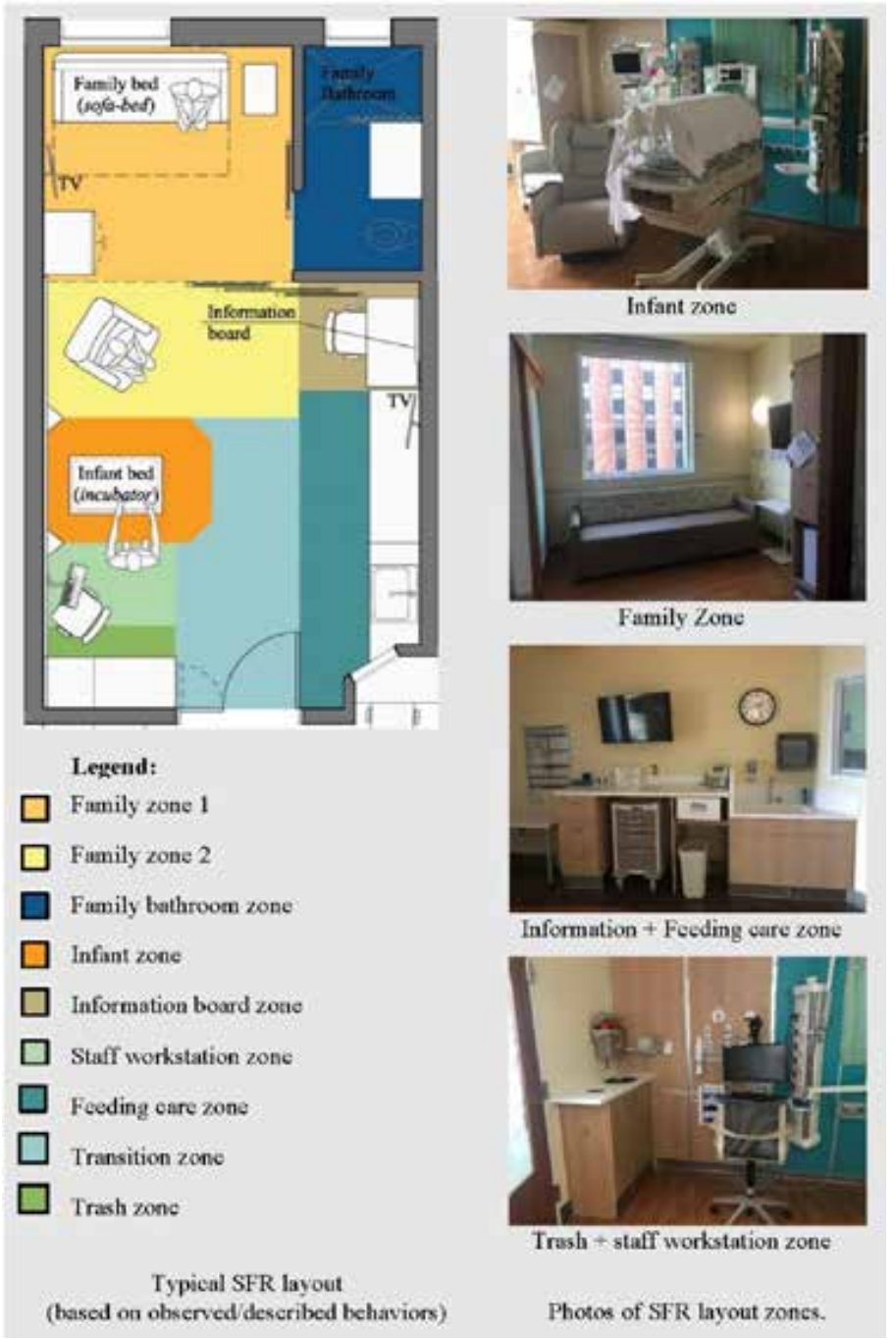

Figure 2: Floor plan and photos illustrating functional zones in a typical SFR.

The family mentioned the visibility between spaces as supportive to their comfort and reassurance, respectively in the form of window views into the atrium and window views between the SFR and staff workstations near their room. Additionally, the "kid-friendly" aesthetics (artwork and signage), as well as the "vibrant" and "uplifting" wall 
colors found in corridors and SFRs, were perceived by both family and staff as supportive to family comfort (See Figure 3).

At the room level, the presence of the SFR and the couplet-care SFR was unanimously perceived by family and staff as a supporting factor to family prolonged presence at the bedside, family privacy and comfort, and daily living activities, which include childcare. These private rooms were also perceived as supportive to familystaff communication, family education, and caregiving interactions. Conversely, staff sometimes associated the SFR with family seclusion and potential sleep disruptions caused by staff's constant in-and-out flow. This, however, may have been affected by other factors related to family and staff individual attitudes and by staff's dual and somewhat conflicting role of being a care provider to both family and infants, at the same time and environment.

The size of the SFR (average area of 350 square feet) was perceived by family and staff as "more than enough" for their actions and interactions in the room. SFR layout was perceived by staff as even conducive to infection control education due to the sink and vertical headwalls used to demarcate 'clean' and 'dirty' areas in the room. Horizontal surfaces, in turn, were described as insufficient in some SFRs and taken by staff as helpful to maintain clean areas (e.g., countertops, where infant milk is prepared) free of family's potentially contaminated personal items. Furthermore, decorations (e.g., Christmas tree), lighting, and sources of distractions in the SFR (e.g., TV) were perceived by family and staff as supporting family comfort (TVs and decorations acting as "connections to the outside world") and caregiving (lights tailored to moments of family-infant bonding).

"The size of the SFR (average area of 350 square feet) was perceived by family and staff as "more than enough" for their actions and interactions in the room. SFR layout was perceived by staff as even conducive to infection control education due to the sink and vertical headwalls used to demarcate 'clean' and 'dirty' areas in the room. Horizontal surfaces, in turn, were described as insufficient in some SFRs and taken by staff as helpful to maintain clean areas (e.g., countertops, where infant milk is prepared) free of family's potentially contaminated personal items"

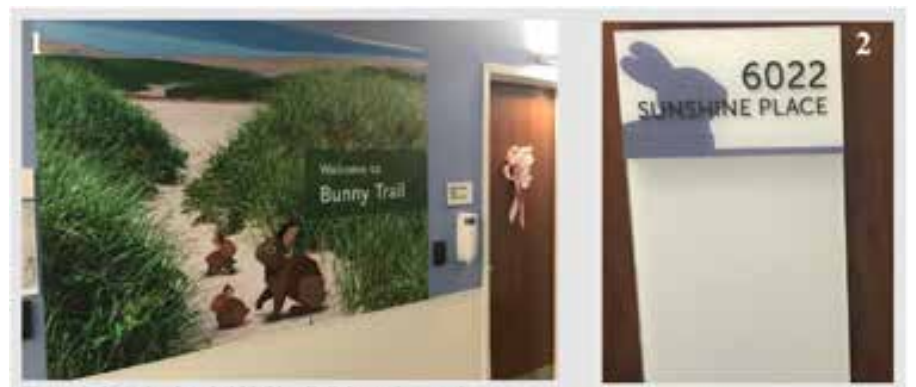

Fhowes 1-2: Animal and "kid-fricndly" themod artwook

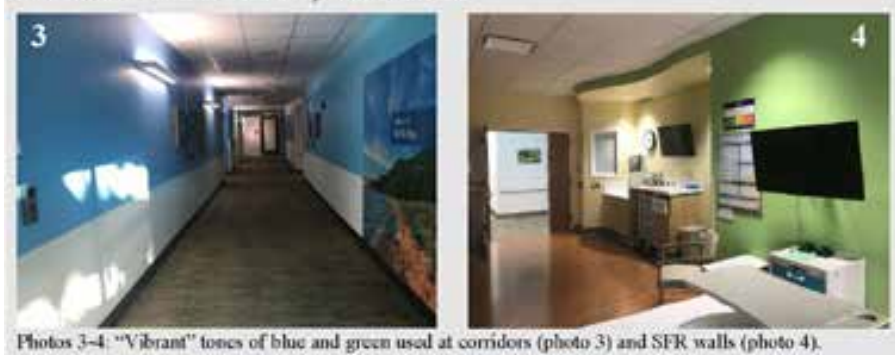

Photos 34: "Vibrant" toncs of bluc and green usol at comidors (photo 3) and SFR walls (photo 4).

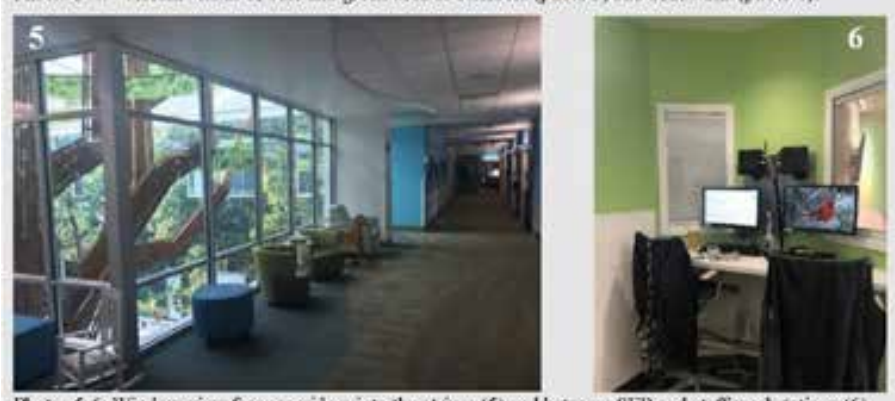

Pholos 5.6: Window view from corridors into the atrium (5) and between SFR and staff worksiations (6)

Figure 3: Photos illustrating aesthetics and visibility factors mentioned by study participants.

Staff and family consistently perceived the information board as a supportive communication interface between family and staff. In contrast, infant milk-related equipment (e.g., milk refrigerator and warmer) were perceived as supportive to family caregiving due to their autonomy and ownership of infant feeding activities. Both family and staff agreed that SFR family zone doors and curtains supported family privacy, and therefore their visual comfort during daily living and caregiving activities. However, the glass material found on family zone and bathroom doors sometimes hindered privacy and family sleep due to light borrowed from the rest of the room. Also, multiple study participants mentioned the lack of ergonomic comfort provided by the family bed, which hindered family sleep comfort and was perceived as one of the reasons why families sometimes may prefer to sleep at the nearby outsourced lodging facility.

Characteristics of the interior of family support spaces also emerged as impacting family presence at the NICU and family behaviors related to daily living and information exchanges. While

NEONATOLOGY TODAY is interested in publishing manuscripts from Neonatologists, Fellows, NNPs and those involved in caring for neonates on case studies, research results, hospital news, meeting announcements, and other pertinent topics.

Please submit your manuscript to: LomaLindaPublishingCompany@gmail.com 
data demonstrated the family lounge being used and appreciated, observations showed that the room is mainly used for quick inand-out daily living activities where families mostly occupied the kitchen area or used the restroom accessed through the room. Unscheduled and informal socialization between families was rarely observed in the room. The presence of the meeting room, on the other hand, was perceived by staff as supporting familyto-family socialization and family discharge education, where features such as storage and equipment (e.g., car-seat replica) supported teaching ergonomics.

\section{Design Recommendations for Family Engagement in the NICU}

While contemporary NICU design guidelines $(20,21)$ suggest room size and zoning standards (e.g. family, patient, and staff zone distinctions inside the SFR), as well as the inclusion of specific types of spaces in the NICU (e.g., library and family lounge), empirical data provided by this study support recommendation of NICU design for family engagement that are more evidence-based. First and foremost, our findings support adopting the SFR NICU design model as a resource to support family engagement. Potential challenges presented by the presence of the SFR to family seclusion may be mitigated with shared and adequately sized family support spaces with activities inside the unit rather than outside, and in close proximity and easy accessibility to SFRs, as to support family-to-family and family-staff informal interactions within infant proximity. We also recommend the integration of private bathrooms adjacent to SFRs and inpatient mother accommodations in the room (couplet-care) to facilitate the family's prolonged bedside presence and wellbeing. Additionally, we found the deployment of bright colors and infant-like decorations across the unit to be successful.

More specific design recommendations relate to interior design features of SFRs and family support rooms. In the SFR, the room layout should translate to a spatial hierarchy that facilitates different locations of privacy-sensitive family behaviors observed in the room. While family zone doors and curtains contribute to shielding families during activities conducted away from infants (e.g., getting dressed and pumping breast milk), other activities like breastfeeding and skin-to-skin care may need additional elements to protect them visually at the bedside while also securing staffto-infant supervision. This may be challenging but alleviated by additional shields or by layout or shape changes in the room. The physical arrangement of the room should also afford direct visibility between family, staff, and infant, and between family, staff, and information displays, facilitating family-infant supervision, familystaff communication, and information access and awareness for family members. In the family zone, family presence can be increased by providing more comfortable beds, sources of positive distractions, family storage, and space for artwork and decorations. Moreover, providing enough sitting in the SFR is likely to facilitate interactions with staff at eye level when family members are using the kangaroo chair.

Due to different levels of privacy needed during social interactions occurring in family support rooms, layout flexibility is also recommended in the design of NICU spaces with a shared kitchen, living room, and play areas. This can be achieved by providing mobile furniture and vertical partitions that can adapt to different group sizes involved in social interactions and to different levels of personal space desired by family members. Spatial hierarchy can be created by furniture or wall positioning to allow for different activities and people to coexist in these shared spaces (e.g., children coexisting with adults, family events coexisting with daily living activities), affording choice for families according to their preferred interaction in the room (e.g., alone time versus socialization). Aiming for balancing family-infant proximity and an increased sense of community in the NICU, findings from this study also recommend sources of distraction in family support rooms, such as TVs and childcare distractions, therefore supporting family respite, socialization, and childcare.

\section{"By evaluating the design of an SFR NICU designed to promote family engagement, this research was able to show the combined impact of unit layout and aesthetics, SFRs, and family support rooms on family presence, care, information exchanges, and caregiving, from the perspectives of both family and staff. We demonstrated how SFR affords privacy as a key environmental quality to facilitate family presence, welllbeing, in- depth family-staff interactions, and intimate family-infant interactions."}

\section{Conclusions}

By evaluating the design of an SFR NICU designed to promote family engagement, this research was able to show the combined impact of unit layout and aesthetics, SFRs, and family support rooms on family presence, care, information exchanges, and caregiving, from the perspectives of both family and staff. We demonstrated how SFR affords privacy as a key environmental quality to facilitate family presence, wellbeing, in-depth family-staff interactions, and intimate family-infant interactions. This study helps guide future NICU projects by providing them empirical rather than best practice design recommendations. This study adds to the body of knowledge around NICU design by emphasizing and exploring in-depth the perspective of family needs, thereby contributing to existing best practices literature such as the $\mathrm{FGI}$ Guidelines and the Journal of Perinatology Recommended Standards for Newborn ICU Design.

\section{References:}

1. Stephens BE, Tucker $R$, Vohr BR. Special health care needs of infants born at the limits of viability. Pediatrics. 2010;125(6):1152-1158. doi:10.1542/peds.2009-1922

2. Fenwick J, Barclay L, Schmied V. Craving closeness: a grounded theory analysis of women's experiences of mothering in the Special Care Nursery. Women Birth. 2008;21(2):71-85. doi:10.1016/j.wombi.2008.03.006

3. Ortenstrand A, Westrup B, Broström EB, et al. The Stockholm Neonatal Family Centered Care Study: effects on length of stay and infant morbidity. Pediatrics. 2010;125(2):e278- 
e285. doi:10.1542/peds.2009-1511

4. Altimier LB., Eichel M., Warner B, et al. Developmental care: Changing the NICU physically and behaviorally to promote patient outcomes and contain costs. Neonatal Intensive Care. 2005; 18(4), 12-16.

5. Welch CD, Check J, O'Shea TM. Improving care collaboration for NICU patients to decrease length of stay and readmission rate. BMJ Open Qual. 2017;6(2):e000130. Published 2017 Oct 21. doi:10.1136/bmjoq-2017-000130

6. Carman KL, Dardess $P$, Maurer M, et al. Patient and family engagement: a framework for understanding the elements and developing interventions and policies. Health Aff (Millwood). 2013;32(2):223-231. doi:10.1377/h/thaff.2012.1133

7. Shepley MM. Design for pediatric and neonatal critical care. Routledge; 2014.

8. Bosch S, Bledsoe T, Jenzarli A. Staff Perceptions Before and After Adding Single-Family Rooms in the NICU. HERD. 2012;5(4):64-75. doi:10.1177/193758671200500406

9. Domanico R, Davis DK, Coleman F, Davis BO. Documenting the NICU design dilemma: comparative patient progress in open-ward and single family room units. J Perinatol. 2011;31(4):281-288. doi:10.1038/jp.2010.120

10. Cone SK, Short S, Gutcher G. From "baby barn" to the "single family room designed NICU": A report of staff perceptions one year post occupancy. Newborn and Infant Nursing Reviews. 2010; 10(2), 97-103.

11. Ulrich $R S$, Zimring $C$, Zhu $X$, et al. A review of the research literature on evidence-based healthcare design. HERD. 2008;1(3):61-125. doi:10.1177/193758670800100306

12. White RD. Designing environments for developmental care. Clin Perinatol. 2011;38(4):745-749. doi:10.1016/j. clp.2011.08.012

13. Shahheidari M, Homer C. Impact of the design of neonatal intensive care units on neonates, staff, and families: a systematic literature review. J Perinat Neonatal Nurs. 2012;26(3):260-268. doi:10.1097/JPN.0b013e318261ca1d

14. Lawhon G. Facilitation of parenting the premature infant within the newborn intensive care unit. J Perinat Neonatal Nurs. 2002;16(1):71-82. doi:10.1097/00005237-20020600000008

15. Coleman V, Smith L, Bradshaw M (Eds.). Family-centered Care: Concept, Theory and Practice. Palgrave; 2002.

16. Brazy JE, Anderson BM, Becker PT, Becker M. How parents of premature infants gather information and obtain support. Neonatal Netw. 2001;20(2):41-48. doi:10.1891/07300832.20.2.41

17. Bramwell R, Weindling M; FVWR Research Team. Families' views on ward rounds in neonatal units. Arch Dis Child Fetal Neonatal Ed. 2005;90(5):F429-F431. doi:10.1136/ adc. 2004.061168

18. White R, Whitman T. Design of ICUs. Pediatrics. 1992; 89(6), 1267-1267.

19. Olding M, McMillan SE, Reeves S, Schmitt MH, Puntillo K, Kitto S. Patient and family involvement in adult critical and intensive care settings: a scoping review. Health Expect. 2016;19(6):1183-1202. doi:10.1111/hex.12402

20. The Facility Guidelines Institute. Guidelines for design and construction of hospitals and outpatient facilities. Chicago, IL: American Society for Healthcare Engineering of the American Hospital Association; 2018.

21. White RD, Smith JA, Shepley MM; Committee to Establish
Recommended Standards for Newborn ICU Design. Recommended standards for newborn ICU design, eighth edition. J Perinatol. 2013;33 Suppl 1:S2-S16. doi:10.1038/jp.2013.10

Conflict of Interest Disclosure: ZGF Architects funded data collection costs for this research
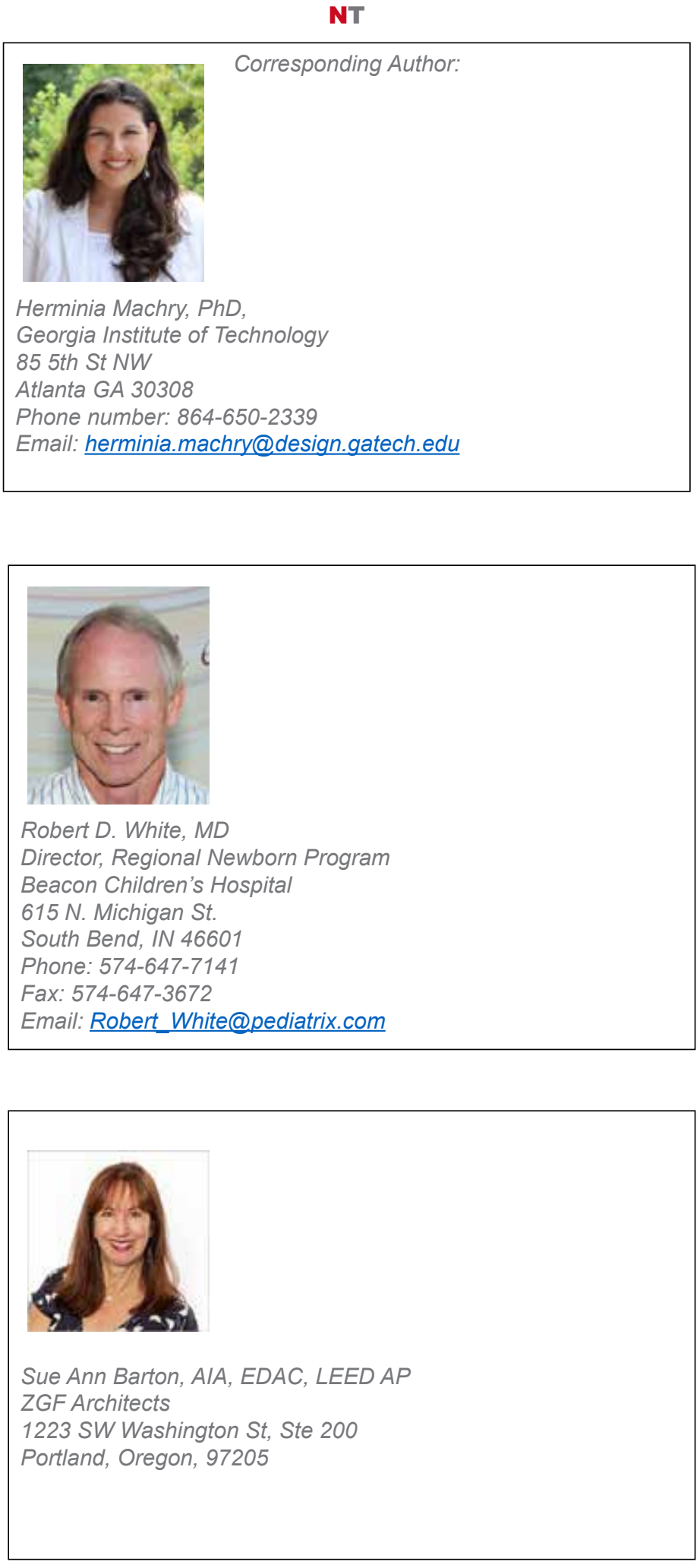